\title{
Mapping tori of free group automorphisms, and the Bieri-Neumann-Strebel invariant of graphs of groups
}

\author{
Christopher H. Cashen and Gilbert Levitt \\ Communicated by Linus Kramer
}

\begin{abstract}
Let $G$ be the mapping torus of a polynomially growing automorphism of a finitely generated free group. We determine which epimorphisms from $G$ to $\mathbb{Z}$ have finitely generated kernel, and we compute the rank of the kernel. We thus describe all possible ways of expressing $G$ as the mapping torus of a free group automorphism. This is similar to the case for 3-manifold groups, and different from the case of mapping tori of exponentially growing free group automorphisms. The proof uses a hierarchical decomposition of $G$ and requires determining the Bieri-Neumann-Strebel invariant of the fundamental group of certain graphs of groups.
\end{abstract}

\section{Introduction}

Given an automorphism $\alpha$ of a group $F$, one may form its mapping torus

$$
G_{\alpha}=F \rtimes_{\alpha} \mathbb{Z}=\left\langle F, t \mid t^{-1} f t=\alpha(f)\right\rangle
$$

and obtain an exact sequence $1 \rightarrow F \rightarrow G_{\alpha} \rightarrow \mathbb{Z} \rightarrow 1$. Geometrically, any fibration over the circle leads to such an exact sequence, with $F$ the fundamental group of the fiber and $\alpha$ induced by the monodromy. If the fiber is compact, then $F$ and $G_{\alpha}$ are finitely generated.

Conversely, any epimorphism $\varphi: G \rightarrow \mathbb{Z}$ yields a split exact sequence

$$
1 \rightarrow \operatorname{ker} \varphi \rightarrow G \rightarrow \mathbb{Z} \rightarrow 1,
$$

but in general $G$ finitely generated does not imply that $\operatorname{ker} \varphi$ is finitely generated. We will say that $\varphi: G \rightarrow \mathbb{Z}$ (or any multiple of $\varphi$ ) comes from a fibration if $\operatorname{ker} \varphi$ is finitely generated. We then call $F=\operatorname{ker} \varphi$ the fiber, and we define the

First author partially supported by European Research Council (ERC) grant \#259527 'ANALYTIC' of G. Arzhantseva and the Austrian Science Fund (FWF) M1717-N25. Both authors partially supported by Agence Nationale de la Recherche (ANR) grant ANR-2010-BLAN-116-01 GGAA. They also thank the Erwin Schrödinger Institute workshop "Geometry of Computation in Groups".

(ब) BY This article is distributed under the terms of the Creative Commons Attribution 3.0 Public License. 
monodromy as the element $\Phi \in \operatorname{Out}(F)$ determined by the action of the generator of $\mathbb{Z}$. The group $G$ is isomorphic to the mapping torus $G_{\alpha}$ of any $\alpha \in \operatorname{Aut}(F)$ representing $\Phi$.

With this terminology, one may ask, given $G$, which maps $\varphi: G \rightarrow \mathbb{Z}$ come from a fibration. This was answered by Thurston [31] when $G$ is a 3-manifold group, or equivalently when the fiber is a surface group. The question is more subtle when $F$ is a free group. Indeed, the class of free-by-cyclic groups remains largely mysterious, in spite of results such as $[6,11,14]$.

Recent work by Dowdall, Kapovich, and Leininger $[9,10]$ studies mapping tori of exponentially growing automorphisms of free groups. We study the opposite case, when monodromies are polynomially growing automorphisms.

Theorem 1.1 (see Theorems 5.2 and 6.1). Let $G=G_{\alpha}=\mathbb{F}_{n} \rtimes_{\alpha} \mathbb{Z}$ for $\alpha \in \operatorname{Aut}\left(\mathbb{F}_{n}\right)$ polynomially growing, with $n \geq 2$. There exist elements $t_{1}, \ldots, t_{n-1}$ in $G$ (not necessarily distinct), and $k \geq 1$, such that, given an epimorphism $\varphi: G \rightarrow \mathbb{Z}$, the following hold:

- If some $\varphi\left(t_{i}\right)$ is 0 , then $\operatorname{ker} \varphi$ virtually surjects onto $\mathbb{F}_{\infty}$ (an infinitely generated free group).

- If no $\varphi\left(t_{i}\right)$ is 0 , then $\operatorname{ker} \varphi$ is a finitely generated free group whose rank is

$$
r=1+\frac{1}{k} \sum_{i=1}^{n-1}\left|\varphi\left(t_{i}\right)\right| .
$$

Freeness of $\operatorname{ker} \varphi$ when finitely generated was proved in [15, Remark 2.7]. Any $\varphi$ such that no $\varphi\left(t_{i}\right)$ is 0 expresses $G$ as the mapping torus of an automorphism of a finitely generated free group. It is known [24] that this automorphism is polynomially growing, with the same degree of growth as the original $\alpha$.

It follows from the theorem that any homomorphism $\varphi: G \rightarrow \mathbb{R}$ may be approached by fibrations, and $\operatorname{ker} \varphi$ virtually maps onto $\mathbb{F}_{\infty}$ if it is not finitely generated. We will also see that $G$ cannot be written as the mapping torus of an injective, non-surjective, endomorphism of a finitely generated free group. None of these properties holds for mapping tori of arbitrary automorphisms of free groups.

We prove Theorem 1.1 by induction, using the fact that $G$ admits a hierarchy: it may be iteratively split along cyclic groups until vertex groups are $\mathbb{Z}^{2}$. The inductive step requires us to understand fibrations of fundamental groups of graphs of groups. We do so in the more general context of the Bieri-Neumann-Strebel (BNS) invariant.

Recall $[3,29]$ that the BNS invariant $\Sigma(G)$ (or $\Sigma^{1}(G)$, in the notation of [29]) of a finitely generated group $G$ is a certain open subset of the sphere $S(G)$ of projective classes $[\varphi]$ of non-zero homomorphisms $\varphi: G \rightarrow \mathbb{R}$. The connection with 
the previous discussion is that $\varphi: G \rightarrow \mathbb{Z}$ is a fibration if and only if $\Sigma(G)$ contains $[\varphi]$ and $[-\varphi]$.

We investigate the BNS invariant of graphs of groups in Section 2. So far, no systematic such study has appeared in print; after receiving a preliminary version of this paper, Ralph Strebel sent us the unpublished monograph [4], whose Theorem II.5.1 is very similar to our results.

Say that a graph of groups is reduced if no edge carries a trivial amalgam $A *_{A} B$, and define the class $\varsigma$ as consisting of all finitely generated groups $H$ such that $\Sigma(H)=S(H)$ (this condition is equivalent to $[H, H]$ being finitely generated).

Theorem 1.2 (Corollaries 2.9 and 2.10). Let $G$ be the fundamental group of a finite reduced graph of groups $\Gamma$, with $G$ finitely generated. Assume that $\Gamma$ is not an ascending HNN-extension. Consider a non-zero map $\varphi: G \rightarrow \mathbb{R}$.

- If edge groups are in $\varsigma$, then $[\varphi] \in \Sigma(G)$ if and only if $\varphi$ is non-trivial on every edge group and $\left[\varphi_{\mid G_{v}}\right] \in \Sigma\left(G_{v}\right)$ for every vertex group $G_{v}$.

- If vertex groups are in $\varsigma$, then $[\varphi] \in \Sigma(G)$ if and only if $\varphi$ is non-trivial on every edge group. In particular, $\Sigma(G)=-\Sigma(G)$ is the complement of a finite number of rationally defined subspheres.

The second assertion applies in particular to graphs of virtually polycyclic groups. In Section 3 we specialize it to $\mathrm{GBS}_{n}$ groups, defined as fundamental groups of finite graphs of groups with every vertex and edge group isomorphic to $\mathbb{Z}^{n}$. If $G$ is a non-solvable $\mathrm{GBS}_{n}$ group, then $[\varphi] \in \Sigma(G)$ if and only if $\varphi(H)$ is not 0 , where $H$ is any edge group. When $G$ is unimodular (i.e. virtually $\mathbb{Z}^{n} \times \mathbb{F}_{k}$ for some $k \geq 2$ ), or $n=1$, this is equivalent to $\varphi(Z) \neq 0$ with $Z$ the center of $G$.

In Section 4 we extend Theorem 1.2 further to hierarchies, and we study the isomorphism type of $\operatorname{ker} \varphi$ through its action on trees. We apply these results in Section 5 to compute the Bieri-Neumann-Strebel invariant for mapping tori of polynomially growing free group automorphisms: with the notations of Theorem 1.1, a non-zero $\varphi: G \rightarrow \mathbb{R}$ represents an element of $\Sigma(G)$ if and only if no $\varphi\left(t_{i}\right)$ equals 0 , so $\Sigma(G)$ is the complement of a finite union of codimension 1 spheres.

In Section 6 we compute the rank of $\operatorname{ker} \varphi$ when it is finitely generated, thus completing the proof of Theorem 1.1. Another approach to this computation is to use the Alexander norm (see the discussion in [9, Section 1.6]).

In Section 7 we consider mapping tori of finite order automorphisms of free groups $\mathbb{F}_{n}$, with $n \geq 2$. These groups are exactly the non-solvable GBS 1 groups having a non-trivial center [23] (a GBS ${ }_{1}$ group with trivial center has $\Sigma(G)$ empty). Given such a $G$, we determine for which values of $n$ and $k$ one can view $G$ 
as the mapping torus of an element of order $k$ in $\operatorname{Out}\left(\mathbb{F}_{n}\right)$, and also for which $n, k$ there is a subgroup $G_{0} \subset G$ of index $k$, isomorphic to $\mathbb{F}_{n} \times \mathbb{Z}$, and containing the center of $G$. In particular, we show that the set of ranks of fibers is an arithmetic progression.

\section{The BNS invariant of a graph of groups}

We first recall the definition and some properties of the BNS invariant $\Sigma(G)$. See [3] and [29] for details.

Given a finitely generated group $G$, let $S(G)$ be the sphere consisting of projective classes of non-zero homomorphisms $\varphi: G \rightarrow \mathbb{R}$. We write $[\varphi]$ for the class of $\varphi$, so that $[\varphi]=[\psi]$ if and only if $\varphi=\lambda \psi$ with $\lambda>0$. The dimension of $S(G)$ is $b_{1}(G)-1$, with $b_{1}(G)$ the first Betti number. If $X \subset \Sigma(G)$, let $-X$ be the set of classes $[-\varphi]$ for $[\varphi] \in X$.

A class $[\varphi] \in S(G)$ is discrete if every homomorphism in the class has discrete image, which is equivalent to saying that $[\varphi]$ contains a surjection onto $\mathbb{Z}$.

For $H \subset G$, let $S(G, H)$ denote the subsphere $\{[\varphi] \in S(G) \mid H \subset \operatorname{ker} \varphi\}$. Its complement in $S(G)$ is denoted $S(G, H)^{c}$. Let $\iota_{H}: H \hookrightarrow G$ denote the inclusion map, and let $\iota_{H}^{*}: S(G, H)^{c} \rightarrow S(H)$ be the restriction map $[\varphi] \mapsto\left[\left.\varphi\right|_{H}\right]$.

Let $\operatorname{Cay}(G, \mathcal{E})$ be the Cayley graph of $G$ with respect to a finite generating set $\mathcal{G}$. We identify its vertex set with $G$. Given $\varphi: G \rightarrow \mathbb{R}$, let $\operatorname{Cay}(G, \mathcal{E})^{[0, \infty)}$ denote the induced subgraph of $\operatorname{Cay}(G, \mathcal{E})$ containing the vertices in $\varphi^{-1}([0, \infty))$. One of the several equivalent definitions of $\Sigma(G)$, see [29], is that $[\varphi] \in S(G)$ belongs to $\Sigma(G)$ if and only if $\operatorname{Cay}(G, \mathcal{E})^{[0, \infty)}$ is connected for some, equivalently, for every, finite generating set $\mathscr{E}$ of $G$. The set $\Sigma(G)$ is open in $S(G)$. See $[3,7,21,26,29]$ for alternate definitions.

In [3, Theorem B1] the following is stated: if $N \triangleleft G$, with $G / N$ abelian, then $N$ is finitely generated if and only if $S(G, N) \subset \Sigma(G)$. Applied to $N=[G, G]$, this shows that $\Sigma(G)=S(G)$ if and only if $[G, G]$ is finitely generated. When $N=\operatorname{ker} \varphi$, with $\varphi: G \rightarrow \mathbb{Z}$, then $S(G, N)=\{[\varphi],-[\varphi]\}$, so $\operatorname{ker} \varphi$ is finitely generated for discrete $[\varphi]$ if and only if both $[\varphi]$ and $-[\varphi]$ are in $\Sigma(G)$.

As shown in [3, Corollary F], results of Thurston [31] imply that, if $G$ is the fundamental group of a compact 3-manifold, then $\Sigma(G)=-\Sigma(G)$ and is a disjoint union of finitely many open convex rational polyhedra in $S(G)$.

By a splitting of a group $G$, we will mean a one-edge splitting, i.e. a decomposition of $G$ as an amalgam $G_{1} *_{A} G_{2}$ or an HNN-extension $G_{1} *_{A}$. We denote by $\iota_{G_{i}}$ the natural inclusion from $G_{i}$ into $G$.

The following lemma says that, if $\varphi: G \rightarrow \mathbb{R}$ does not vanish on $A$, and its restriction to each $G_{i}$ represents an element of $\Sigma\left(G_{i}\right)$, then $[\varphi]$ is in $\Sigma(G)$. 
Lemma 2.1. The following statements hold.

(1) Let $G$ be an amalgamated free product $G_{1} *_{A} G_{2}$, with $G_{1}, G_{2}$ finitely generated. Then

$$
S(G, A)^{c} \cap\left(\iota_{G_{1}}^{*}\right)^{-1}\left(\Sigma\left(G_{1}\right)\right) \cap\left(\iota_{G_{2}}^{*}\right)^{-1}\left(\Sigma\left(G_{2}\right)\right) \subset \Sigma(G) .
$$

(2) Let $G$ be an HNN-extension $\left\langle G_{1}, t\right| t^{-1} a t=\sigma(a)$ for $\left.a \in A\right\rangle$ with finitely generated base group $G_{1}$, subgroup $A<G_{1}$, and injection $\sigma: A \hookrightarrow G_{1}$. Then

$$
S(G, A)^{c} \cap\left(\iota_{G_{1}}^{*}\right)^{-1}\left(\Sigma\left(G_{1}\right)\right) \subset \Sigma(G) .
$$

Proof. Case (1) is an immediate corollary of [29, Lemma B1.14]. We prove case (2), which is very similar. Assume $[\varphi] \in S(G, A)^{c} \cap\left(\iota_{G_{1}}^{*}\right)^{-1}\left(\Sigma\left(G_{1}\right)\right)$.

Take a finite generating set for $G_{1}$ and append $t$ to get a finite generating set for $G$. Consider the corresponding Cayley graph. Since $[\varphi] \in\left(\iota_{G_{1}}^{*}\right)^{-1}\left(\Sigma\left(G_{1}\right)\right)$, it follows trivially from the definitions that for every $g \in G$ and $b_{1}, b_{2}$ in $G_{1}$ there exists a path from $g b_{1}$ to $g b_{2}$ in $g G_{1}$ such that every vertex $x$ along the path satisfies $\varphi(x) \geq \min \left(\varphi\left(g b_{1}\right), \varphi\left(g b_{2}\right)\right)$.

An element $g \in G$ can be expressed $g=b_{0} t^{\varepsilon_{1}} b_{1} \cdots t^{\varepsilon_{m}} b_{m}$ with $\varepsilon_{i}= \pm 1$ and $b_{i} \in G_{1}$. The minimal such $m$ is called the syllable length of $g$. We prove by induction on the syllable length that if $\varphi(g) \geq 0$, then there exists a $\varphi$-non-negative path from 1 to $g$, i.e. a path such that every vertex $x$ on the path satisfies $\varphi(x) \geq 0$. This claim implies $[\varphi] \in \Sigma(G)$.

The claim is true for syllable length 0 by the hypothesis that $\left[\left.\varphi\right|_{G_{1}}\right] \in \Sigma\left(G_{1}\right)$. Now suppose $g$ has syllable length $m>0$ and $\varphi(g) \geq 0$, and suppose that the claim is true for shorter syllable length. Let

$$
g=b_{0} t^{\varepsilon_{1}} b_{1} \cdots t^{\varepsilon_{m}} b_{m} \quad \text { and } \quad g^{\prime}=b_{0} t^{\varepsilon_{1}} b_{1} \cdots t^{\varepsilon_{m-1}} b_{m-1} .
$$

Since $[\varphi] \notin S(G, A)$, we can choose $a \in A$ such that

$$
\varphi(a) \geq \max \left(-\varphi\left(g^{\prime}\right),-\varphi\left(g^{\prime} t^{\varepsilon_{m}}\right)\right) .
$$

If $\varepsilon_{m}=1$, then $g=g^{\prime} a a^{-1} t b_{m}=g^{\prime} a t \sigma\left(a^{-1}\right) b_{m}$, where $\varphi\left(g^{\prime} a\right)$ and $\varphi\left(g^{\prime} a t\right)$ are non-negative and $g^{\prime} a$ has syllable length $<m$. By the induction hypothesis there is a $\varphi$-non-negative path from 1 to $g^{\prime} a$. Concatenating the $t$-edge gives a $\varphi$-non-negative path from 1 to $g^{\prime} a t$. Finally, the remark of the second paragraph implies that we can concatenate this path with a path in $g^{\prime}$ at $G_{1}$ from $g^{\prime} a t$ to $g=g^{\prime}$ at $\sigma\left(a^{-1}\right) b_{m}$ to get a $\varphi$-non-negative path from 1 to $g$.

If $\varepsilon_{m}=-1$, the argument is similar using the relation $g=g^{\prime} t^{-1} a a^{-1} b_{m}=$ $g^{\prime} \sigma(a) t^{-1} a^{-1} b_{m}$ and the fact that $\varphi(\sigma(a))$ equals $\varphi(a)$. The path passes through $g^{\prime} \sigma(a)$ and $g^{\prime} \sigma(a) t^{-1}$. 
Corollary 2.2. Let $G$ be the fundamental group of a finite graph of groups, with every vertex group finitely generated. Let $\varphi: G \rightarrow \mathbb{R}$ be a homomorphism. Assume:

(1) $\varphi$ is non-trivial on every edge group,

(2) $\left[\varphi_{\mid G_{v}}\right]$ belongs to $\Sigma\left(G_{v}\right)$ for every vertex group $G_{v}$.

Then $[\varphi] \in \Sigma(G)$.

Proof. By induction on the number of edges, writing $G$ as an amalgam or an HNN-extension of groups which are fundamental groups of graphs of groups with fewer edges.

We now study the converse, so we assume $[\varphi] \in \Sigma(G)$ and we consider restrictions of $\varphi$ to edge and vertex groups. To deduce (1), we simply need some non-degeneracy assumptions.

Definition 2.3. A graph of groups $\Gamma$ is said to be reduced if, given an edge $e$ with distinct endpoints $v_{1}, v_{2}$, the inclusions $G_{e} \hookrightarrow G_{v_{i}}$ are proper.

In particular, an $\mathrm{HNN}$-extension is always reduced. An amalgam $G_{1} *_{A} G_{2}$ is reduced if and only if it is non-trivial $\left(A \neq G_{1}, G_{2}\right)$.

If $\Gamma$ is not reduced, we may make it reduced by iteratively collapsing edges making it non-reduced. This does not introduce new vertex groups since the group $G_{v_{1}} *_{v_{v_{1}}} G_{v_{2}}$ is isomorphic to $G_{v_{2}}$.

Definition 2.4. An HNN-extension $G=\left\langle G_{1}, t\right| t^{-1} a t=\sigma(a)$ for $\left.a \in A\right\rangle$ is said to be ascending if $A=G_{1}$ and descending if $\sigma(A)=G_{1}$. It is strictly ascending if it is ascending and not descending.

Replacing the stable letter $t$ by its inverse reverses ascending/descending. We say that a graph of groups $\Gamma$ is not an ascending HNN-extension if it is not an $\mathrm{HNN}$-extension (it has more than one edge or more than one vertex), or it is an HNN-extension but both $A$ and $\sigma(A)$ are proper subgroups of $G_{1}$.

Proposition 2.5. Let $G$ be the fundamental group of a finite reduced graph of groups $\Gamma$, with $G$ finitely generated. Assume that $\Gamma$ is not an ascending $H N N$-extension. If $[\varphi] \in \Sigma(G)$, then $\varphi$ is non-trivial on every edge group.

Proof. The special case when $\Gamma$ has a single edge is [29, Proposition C2.13]. Given any edge $e$ of $\Gamma$, collapse all other edges. The resulting graph of groups is not an ascending $\mathrm{HNN}$-extension because $\Gamma$ is reduced, so we may apply the special case to conclude that $\varphi$ is non-trivial on $G_{e}$. 
Remark. The proposition is wrong in the case of an ascending HNN-extension (see Remark 2.11). Rather than assuming that $\Gamma$ is reduced and not an ascending HNN-extension, one could assume that its Bass-Serre tree is minimal and irreducible.

The following example shows that some more serious restriction is necessary in order to deduce the statement $\left[\varphi_{\mid G_{v}}\right] \in \Sigma\left(G_{v}\right)$ (item (2) in Corollary 2.2) from the assumption $[\varphi] \in \Sigma(G)$.

Example 2.6. View $G=\mathbb{F}_{2} \times \mathbb{F}_{2}$ as the amalgam of $\mathbb{F}_{2} \times \mathbb{Z}=\langle a, b\rangle \times\langle c\rangle$ with $\mathbb{F}_{2} \times \mathbb{Z}=\langle a, b\rangle \times\langle d\rangle$ over $\mathbb{F}_{2}=\langle a, b\rangle$. If $\varphi: G \rightarrow \mathbb{R}$ kills $d$ but none of $a, b, c$, then $[\varphi] \in \Sigma(G)$ but $\left[\varphi_{\mid G_{v}}\right] \notin \Sigma\left(G_{v}\right)$ for $G_{v}=\langle a, b\rangle \times\langle d\rangle$ (see [3, Theorem 7.4] or [29, Proposition A2.7] for $\Sigma$ of a direct product). This may be generalized to groups of the form $A \times \mathbb{F}_{2}$ with $\Sigma(A) \neq S(A)$.

Proposition 2.7. Let $G$ be the fundamental group of a finite graph of groups $\Gamma$, with all edge and vertex groups finitely generated. Assume that $[\varphi] \in \Sigma(G)$, and $\left[\varphi_{\mid G_{e}}\right] \in \Sigma\left(G_{e}\right)$ for every edge group $G_{e}$ (in particular, $\varphi\left(G_{e}\right) \neq 0$ ). Then we have $\left[\varphi_{\mid G_{v}}\right] \in \Sigma\left(G_{v}\right)$ for every vertex group $G_{v}$.

Proof. As in the proof of Corollary 2.2, it suffices to prove the result when $\Gamma$ has a single edge: $G=G_{1} *_{A} G_{2}$ or $G=\left\langle G_{1}, t\right| t^{-1} a t=\sigma(a)$ for $\left.a \in A\right\rangle$. Since $\varphi$ does not vanish on $A$, it does not vanish on $G_{i}$, so $\left[\left.\varphi\right|_{G_{i}}\right] \in S\left(G_{i}\right)$.

First suppose $G=G_{1} *_{A} G_{2}$. Fix a finite generating set $\mathcal{A}$ of $A$, and choose finite generating sets $\mathscr{E}_{i}$ for $G_{i}$ extending the respective images of $\mathcal{A}$, with $\mathscr{H}_{i} \backslash \mathcal{A} \subset$ $G_{i} \backslash A$. Their union $\mathscr{E}=\mathscr{G}_{1} \cup \mathscr{E}_{2}$ is a finite generating set for $G$.

Take two vertices $g$ and $h$ in $\operatorname{Cay}\left(G_{1}, \mathscr{E}_{1}\right)^{[0, \infty)}$. We have to join them by a path contained in $\operatorname{Cay}\left(G_{1}, \mathscr{E}_{1}\right)^{[0, \infty)}$.

There exists a path $p$ joining them in $\operatorname{Cay}(G, \mathscr{E})^{[0, \infty)}$, since $[\varphi] \in \Sigma(G)$. Write labels along $p$ as $g_{1} g_{2} \ldots g_{k}$ with $g_{i} \in \mathcal{E}$. If every $g_{i}$ is in $\mathscr{E}_{1}$, then $p$ is contained in $\operatorname{Cay}\left(G_{1}, \mathscr{E}_{1}\right)$ and we are done. Otherwise, by standard facts about free products with amalgamation, there exists a subpath $g_{i+1} \ldots g_{j}$ containing an edge with label in $\mathscr{E}_{2} \backslash \mathcal{A}$ and representing an element of $A$.

Since $\mathcal{E}_{1}$ is an extension of $\mathcal{A}$, and $\operatorname{Cay}(A, \mathcal{A})^{[0, \infty)}$ is connected because $\left[\left.\varphi\right|_{A}\right]$ is an element of $\Sigma(A)$, we may replace the subpath $g_{i+1} \ldots g_{j}$ of $p$ by a subpath contained in $\operatorname{Cay}(A, \mathcal{A})^{[0, \infty)} \subset \operatorname{Cay}\left(G_{1}, \mathscr{E}_{1}\right)^{[0, \infty)}$. This reduces the number of labels of $p$ not in $\mathscr{E}_{1}$.

Repeat this process until all labels of $p$ are in $\mathscr{E}_{1}$. This gives a path from $g$ to $h$ in $\operatorname{Cay}\left(G_{1}, \mathscr{E}_{1}\right)^{[0, \infty)}$, so $\left[\left.\varphi\right|_{G_{1}}\right] \in \Sigma\left(G_{1}\right)$. The same argument applies to $G_{2}$.

In the HNN-extension case, choose a finite generating set $\mathscr{E}_{1}$ of $G_{1}$ extending $\mathcal{A} \cup \sigma(\mathcal{A})$. Let $\mathcal{H}=\mathscr{G}_{1} \cup\{t\}$, and apply the same argument as in the amalgamated product case (using Britton's lemma) to conclude $\left[\left.\varphi\right|_{G_{1}}\right] \in \Sigma\left(G_{1}\right)$. 
Propositions 2.5 and 2.7 do not provide a complete converse of Corollary 2.2, because Proposition 2.5 only guarantees $\varphi\left(G_{e}\right) \neq 0$, not $\left[\varphi_{\mid G_{e}}\right] \in \Sigma\left(G_{e}\right)$. This motivates the following definition.

Definition 2.8. Let $\varsigma$ be the class of finitely generated groups $G$ with the property that $\Sigma(G)=S(G)$.

Equivalently, $\checkmark$ is the class of finitely generated groups with finitely generated commutator subgroup [3].

Recall that a group is slender if every subgroup is finitely generated. Slender groups belong to $\varsigma$. Examples of slender groups include finitely generated virtually abelian groups and virtually polycyclic groups.

Corollary 2.2 and Propositions 2.5 and 2.7 now imply:

Corollary 2.9. Let $G$ be the fundamental group of a finite reduced graph of groups $\Gamma$, with $G$ finitely generated and edge groups in 5 . Assume that $\Gamma$ is not an ascending $H N N$-extension. Then $[\varphi] \in \Sigma(G)$ if and only if $\varphi$ is non-trivial on every edge group and $\left[\varphi_{\mid G_{v}}\right] \in \Sigma\left(G_{v}\right)$ for every vertex group $G_{v}$,

$$
\Sigma(G)=\bigcap_{e \in \mathcal{E} \Gamma} S\left(G, G_{e}\right)^{c} \cap \bigcap_{v \in \mathcal{V} \Gamma}\left(\iota_{G_{v}}^{*}\right)^{-1}\left(\Sigma\left(G_{v}\right)\right) .
$$

Note that all vertex groups are finitely generated, so $\Sigma\left(G_{v}\right)$ is defined.

Corollary 2.10. Let $G$ be the fundamental group of a finite reduced graph of groups $\Gamma$, with every vertex group in 5 . Assume that $\Gamma$ is not an ascending $H N N$-extension. Then $[\varphi] \in \Sigma(G)$ if and only if $\varphi$ is non-trivial on every edge group. In particular,

$$
\Sigma(G)=-\Sigma(G)=\bigcap_{e \in \mathcal{E} \Gamma} S\left(G, G_{e}\right)^{c}
$$

is the complement of a finite number of rationally defined subspheres.

This applies in particular to graphs of virtually polycyclic groups.

Remark 2.11. If the group $G=\left\langle G_{1}, t\right| t^{-1} a t=\sigma(a)$ for $\left.a \in G_{1}\right\rangle$ is an ascending $\mathrm{HNN}$-extension, then $S\left(G, G_{e}\right)$ consists of two points, the projective classes containing $\varphi_{ \pm}: G \rightarrow \mathbb{Z}$ defined by $\varphi\left(G_{1}\right)=0$ and $\varphi(t)= \pm 1$. When $G_{1}$ is finitely generated, $\Sigma(G)$ contains $\left[\varphi_{+}\right]$, and contains $\left[\varphi_{-}\right]$if and only if the extension is not strictly ascending ([3, Proposition 4.4]). In particular, $\Sigma(G) \neq-\Sigma(G)$ if $G$ is a strictly ascending HNN-extension with finitely generated base group.

We thus have the following. 
Corollary 2.12. If $\Sigma(G) \neq-\Sigma(G)$, in particular if $G$ is a strictly ascending $H N N$-extension with finitely generated base group, then every decomposition of $G$ as the fundamental group of a finite reduced graph of groups with vertex groups in $\mathrm{S}$ is a strictly ascending $H N N$-extension.

\section{Generalized Baumslag-Solitar groups}

Let $G$ be as in Corollary 2.10. Assume furthermore that all the inclusions $G_{e} \hookrightarrow G_{v}$ map $G_{e}$ onto a finite index subgroup (this is equivalent to the Bass-Serre tree of $\Gamma$ being locally finite). Then all edge and vertex groups are commensurable (the intersection of any two has finite index in both), so $\Sigma(G)=S(G, H)^{c}$ with $H$ any vertex or edge group. Moreover, a homomorphism $\varphi: G \rightarrow \mathbb{R}$ represents an element of $S(G, H)$ if and only if it factors through the topological fundamental group of $\Gamma$. We thus get:

Corollary 3.1. Let $G$ be as in Corollary 2.10, with all edge and vertex groups commensurable. If $H$ is any vertex or edge group, then $\Sigma(G)=S(G, H)^{c}$ is the complement of a rationally defined subsphere whose dimension is $b-1$, with $b$ the first Betti number of the graph $\Gamma$.

In particular, a rank $n$ generalized Baumslag-Solitar group, or $\mathrm{GBS}_{n}$ group, is the fundamental group $G$ of a finite graph of groups $\Gamma$ all of whose edge and vertex groups are isomorphic to $\mathbb{Z}^{n}$. When $n=1$, we simply say that $G$ is a GBS group.

If $\Gamma$ is an ascending HNN-extension, $G$ is solvable, so we obtain:

Corollary 3.2. If $G$ is a non-solvable $\mathrm{GBS}_{n}$ group, then $\Sigma(G)=S(G, H)^{c}$ with $H$ any edge or vertex group.

In certain cases, we may relate $\Sigma(G)$ to the center $Z(G)$.

Proposition 3.3. Let $G$ be a non-solvable GBS group. Then

$$
\Sigma(G)=S(G, \mathrm{Z}(G))^{c} .
$$

If the center is trivial, $\Sigma(G)=\emptyset$. If not, $\Sigma(G)$ is the complement of a codimension 1 rationally defined subsphere.

The solvable GBS groups are $\mathbb{Z}$ and the Baumslag-Solitar groups

$$
\mathrm{BS}(1, k)=\left\langle a, t \mid t^{-1} a t=a^{k}\right\rangle .
$$

Proof. This follows from [22, Propositions 2.5 and 3.3]. If the center is trivial, edge groups have finite image in the abelianization of $G$ so $\Sigma(G)=\emptyset$. If there is 
a center, it is infinite cyclic, contained in every edge group, and maps injectively into the abelianization.

Going back to arbitrary $n$, suppose that $G=\mathbb{Z}^{n} \times \mathbb{F}_{k}$ for some $k \geq 2$. Then all edge and vertex groups are equal to the $\mathbb{Z}^{n}$ factor, which is the center, and $\Sigma(G)=S(G, Z(G))^{c}$. This may be generalized to $\mathrm{GBS}_{n}$ groups which are virtually $\mathbb{Z}^{n} \times \mathbb{F}_{k}$ (such groups are called unimodular, see below).

Proposition 3.4. If the $\mathrm{GBS}_{n}$ group $G$ is non-solvable and unimodular (i.e. $G$ is virtually $\mathbb{Z}^{n} \times \mathbb{F}_{k}$ with $\left.k \geq 2\right)$, then $\Sigma(G)=S(G, \mathrm{Z}(G))^{c}$.

Proof. Consider the action of $G$ on the Bass-Serre tree $T$ of $\Gamma$ (we may assume that this action is minimal: there is no proper invariant subtree). By a standard argument, the center of $G$ acts as the identity on $T$ (it is elliptic because $T$ is not a line, and its fixed subtree is $G$-invariant), so a $\varphi$ that does not vanish on the center is in $\Sigma(G)$. We have proved $S(G, \mathrm{Z}(G))^{c} \subset \Sigma(G)$ (this also follows from [29, Proposition A2.4]).

Let $G_{0}=\mathbb{Z}^{n} \times \mathbb{F}_{k}$ have finite index in $G$. It acts minimally on $T$, and as above its center $Z\left(G_{0}\right)=\mathbb{Z}^{n}$ acts as the identity on $T$. We deduce that the set of elements of $G$ acting as the identity on $T$ is a normal subgroup $N$ isomorphic to $\mathbb{Z}^{n}$; it has finite index in every edge stabilizer, and contains $Z\left(G_{0}\right)$ with finite index.

If $[\varphi] \in \Sigma(G)$, then $\varphi$ does not vanish on edge stabilizers, so there is an element $x \in Z\left(G_{0}\right)$ with $\varphi(x) \neq 0$. This $x$ has finitely many conjugates in $G$, all contained in the abelian group $N$, and the product of these conjugates is a central element of $G$ which is not killed by $\varphi$. This proves $\Sigma(G) \subset S(G, \mathrm{Z}(G))^{c}$.

We may interpret these results in terms of the modular representation $\Delta$ of $G$. Let $G$ be a non-solvable $\mathrm{GBS}_{n}$ group, and let $G_{e} \cong \mathbb{Z}^{n}$ be an edge group. If $g \in G$, conjugation by $g$ induces an isomorphism

$$
g^{-1} G_{e} g \cap G_{e} \rightarrow G_{e} \cap g G_{e} g^{-1}
$$

between finite index subgroups of $G_{e}$. We define $\Delta(g)$ as the class of this isomorphism in the abstract commensurator of $G_{e}$ (isomorphic to $\mathrm{GL}_{n}(\mathbb{Q})$ ). The groups of Proposition 3.4 are those for which the image $\Delta(G)$ is a finite subgroup of $\mathrm{GL}_{n}(\mathbb{Q})$.

Since $G_{e} \cong \mathbb{Z}^{n}$, we may view $\Delta$ as an action of $G$ on the vector space $G_{e} \otimes \mathbb{Q}$. The codimension of $S\left(G, G_{e}\right)$ is the dimension of the space of invariant vectors for the action on the dual space. The rank of the center of $G$ is the dimension of the space of invariant vectors for the action on the space itself. Unfortunately, these dimensions may be different when $n>1$, so the analogue of Proposition 3.3 can not be true in general. 


\section{Hierarchies}

The goal of this section is to study $\Sigma(G)$, and $\operatorname{ker} \varphi$ for $\varphi: G \rightarrow \mathbb{R}$, when $G$ admits a suitable hierarchical decomposition.

Definition 4.1. A $\mathcal{P}$-hierarchy for a group $G$ is an iterated splitting over subgroups in the class $\mathcal{P}$.

More precisely, a hierarchy may be viewed as a finite rooted tree. Each vertex $\alpha$ carries a group $G(\alpha)$, with the root carrying $G$. If $\alpha$ is not a leaf, it carries a non-trivial splitting of $G(\alpha)$ as an amalgamated product or HNN-extension over a group in the class $\mathcal{P}$, called an edge group of the hierarchy. Moreover, $\alpha$ has one descendant $\beta$ for each factor group in the splitting (so $\alpha$ has one or two descendants), and $G(\beta)$ is the corresponding factor.

The groups carried by the leaves are called the leaf groups of the hierarchy. The number of splittings in the hierarchy is equal to the number of non-leaf vertices.

A $\mathcal{P}$-hierarchy is a good $\mathcal{P}$-hierarchy if all groups $G(\alpha)$ are finitely generated, and no splitting in the hierarchy is an ascending HNN-extension. Recall that $\varsigma$ is the class of finitely generated groups $H$ with $\Sigma(H)=S(H)$.

Theorem 4.2. Suppose $G$ admits a good $\varsigma$-hierarchy, with splittings over subgroups $\left\{A_{i} \mid i \in \mathcal{d}\right\}$, and leaf groups $\left\{H_{j} \mid j \in \mathcal{L}\right\}$. Then

$$
\Sigma(G)=\bigcap_{i \in \mathcal{d}} S\left(G, A_{i}\right)^{c} \cap \bigcap_{j \in \mathcal{A}}\left(\iota_{H_{j}}^{*}\right)^{-1}\left(\Sigma\left(H_{j}\right)\right) .
$$

Proof. The proof is by induction on the number of splittings in the hierarchy. If there are no splittings then $G$ itself is a leaf group and the result is clear. Otherwise, consider the first splitting in the hierarchy, which is of the form $G=G_{1} *_{A} G_{2}$ or $G=G_{1} *_{A}$. Since $A \in \varsigma$ and the splitting is not an ascending HNN-extension, by Corollary 2.9,

$$
\Sigma(G)=S(G, A)^{c} \cap \bigcap_{k \in \mathcal{K}}\left(\iota_{G_{k}}^{*}\right)^{-1}\left(\Sigma\left(G_{k}\right)\right),
$$

where $\mathcal{K}=\{1,2\}$ in the amalgamated product case and $\mathcal{K}=\{1\}$ in the HNN case. For each $k$, consider the vertex carrying $G_{k}$ in the hierarchy for $G$. Its descendant subtree is a hierarchy for $G_{k}$ satisfying the hypotheses of the theorem and having strictly fewer splittings. The theorem then follows by the induction hypothesis.

Corollary 4.3. If $G$ admits a good $\varsigma$-hierarchy with leaf groups in $\varsigma$, then the set $\Sigma(G)=\bigcap_{i \in \mathcal{Q}} S\left(G, A_{i}\right)^{c}$ is the complement of a finite number of rationally 
defined subspheres. In particular, $\Sigma(G)=-\Sigma(G)$, so $G$ does not admit a decomposition as a strictly ascending $H N N$-extension with finitely generated base group (see Remark 2.11).

Theorem 4.4. Suppose $G$ admits a good $\mathbb{Z}$-hierarchy with leaf groups $\left\{H_{j} \mid j \in \mathcal{A}\right\}$ belonging to 5 . Then:

(1) $\Sigma(G)^{c}$ is a finite union of rationally defined subspheres.

(2) If $[\varphi] \in \Sigma(G)$, then $\operatorname{ker} \varphi$ is a free product of groups, each isomorphic to some $\operatorname{ker} \varphi_{\mid H_{j}}$ with $\varphi\left(H_{j}\right) \neq 0$. If $[\varphi]$ is discrete, this is a finite free product.

(3) If $[\varphi] \in \Sigma(G)^{c}$ is discrete, and every group in the hierarchy has first Betti number at least 2 , then $\operatorname{ker} \varphi$ surjects onto $\mathbb{F}_{\infty}$ (an infinitely generated free group).

The first assertion follows from Theorem 4.2. Before proving the others, we need a simple orbit counting lemma:

Lemma 4.5. Let $G$ act transitively on a set $X$. Let $K$ be a normal subgroup, with $p: G \rightarrow G / K$ the quotient map. Let $x \in X$ be arbitrary, with stabilizer $G_{x}$. The number of $K$-orbits in $X$ equals the index of $K G_{X}$ in $G$, and also the index of $p\left(G_{x}\right)$ in $G / K$.

Proof. The group $G$ acts transitively on the set of $K$-orbits, with the stabilizer of $K x$ equal to $K G_{x}$ (which is a subgroup because $K$ is normal). The map $p$ induces a bijection between cosets mod $K G_{x}$ in $G$ and cosets $\bmod p\left(G_{x}\right)$ in $G / K$.

Proof of Theorem 4.4. Assertions (2) and (3) are proved by induction on the number of splittings in the hierarchy. If there are no splittings, then $\Sigma(G)=S(G)$ and the result is clear. Otherwise the first splitting in the hierarchy for $G$ corresponds to either an amalgamated product $G=G_{1} *_{A} G_{2}$ or an HNN-extension $G=G_{1} *_{A}$. The groups $G_{i}$ have shorter hierarchies satisfying the hypothesis of the theorem.

Let $K=\operatorname{ker} \varphi$, so $G / K \simeq \varphi(G)$ is a subgroup of $\mathbb{R}$. We study $K$ through its action on the Bass-Serre tree $T$ of the first splitting in the hierarchy. Since $K$ is normal, vertex stabilizers are conjugate to $K \cap G_{i}=\operatorname{ker} \varphi_{\mid G_{i}}$ for $i=1$ or 2; edge stabilizers are conjugate to $K \cap A=\operatorname{ker} \varphi_{\mid A}$.

Consider the image in $G / K$ of the stabilizer $G_{v}$ of a point $v \in T$ for the action of $G$. It is trivial if $G_{v}<K$ (i.e. if $\varphi\left(G_{v}\right)=0$ ) and infinite otherwise. Applying Lemma 4.5 with $X=G v$, this implies that $G v$ splits into infinitely many $K$-orbits if $\varphi\left(G_{v}\right)=0$. The converse is also true if $[\varphi]$ is discrete: if $\varphi\left(G_{v}\right) \neq 0$, the number of $K$-orbits in $G v$ is the index of $\varphi\left(G_{v}\right)$ in $\varphi(G)$, which is finite since $\varphi(G) \simeq \mathbb{Z}$. In particular, $T / K$ has infinitely many edges if $\varphi(A)=0$, finitely many if $[\varphi]$ is discrete and $\varphi(A) \neq 0$. 
First suppose $\varphi(A) \neq 0$, so $\varphi\left(G_{i}\right) \neq 0$. In this case edge stabilizers for the $K$-action are trivial ( $\varphi$ is injective on $A$ since $A \cong \mathbb{Z}$ ). Thus, $K$ is a free product of groups isomorphic to $\operatorname{ker} \varphi_{\mid G_{i}}$ (and both factors occur in the case of an amalgam). Moreover, if $[\varphi]$ is discrete then $T / K$ is finite, so this is a finite free product.

If $[\varphi] \in \Sigma(G)$, its restrictions to $G_{i}$ belong to $\Sigma\left(G_{i}\right)$ by Corollary 2.9, and the theorem follows by induction. If $[\varphi] \in \Sigma(G)^{c}$, we have $\left[\varphi_{\mid G_{i}}\right] \in \Sigma\left(G_{i}\right)^{c}$ for some $i$. If $[\varphi]$ is discrete and Betti numbers are $\geq 2$, then $\operatorname{ker} \varphi_{\mid G_{i}}$ maps onto $\mathbb{F}_{\infty}$ by induction, and so does $\operatorname{ker} \varphi$.

Now suppose $\varphi(A)=0$. Then $[\varphi] \in \Sigma(G)^{c}$ by Corollary 2.9, so the second assertion of the theorem is proved.

To complete the proof, we construct a surjection from $K$ to $\mathbb{F}_{\infty}$, assuming that $[\varphi]$ is discrete, $\varphi(A)=0$, and Betti numbers are $\geq 2$.

As explained above, $T / K$ has infinitely many edges. If $v$ is a vertex of $T$ with $\varphi\left(G_{v}\right)=0$, the valence of $v$ in $T / G$ is the same as in $T / K$ because $G_{v}$ is conjugate to some $G_{i}$, and the index of $K \cap A$ in $K \cap G_{i}$ equals the index of $A$ in $G_{i}$ since $G_{i} \subset K$.

We distinguish between the amalgamated product and HNN cases.

In the amalgamated product case, if $\varphi\left(G_{1}\right)$ and $\varphi\left(G_{2}\right)$ are both non-zero, there are finitely many vertices in $T / K$ (but infinitely many edges). Viewing $K$ as the fundamental group of the graph of groups $T / K$, we get a surjection from $K$ onto $\mathbb{F}_{\infty}$ by killing all the vertex groups.

If, say, $\varphi\left(G_{1}\right)=0$ (and therefore $\varphi\left(G_{2}\right)=\varphi(G) \neq 0$ ), then $T / K$ is a star with exactly one vertex of type 2 (carrying a conjugate of $G_{2}$ ) and infinitely many valence 1 vertices of type 1 . The groups carried by these terminal vertices are conjugates of $G_{1}$. By hypothesis, $G_{1}$ has first Betti number at least 2 , so there exists a non-zero map from each terminal vertex group to $\mathbb{Z}$ killing the incident edge group (hence also its normal closure). We map $K$ onto $\mathbb{F}_{\infty}$ by killing the central type 2 vertex group, hence all edge groups, and piecing together the surjections to $\mathbb{Z}$ of the resulting quotients of the infinitely many terminal vertices.

In the HNN case, $G \cong\left\langle G_{1}, t \mid t^{-1} A t=\sigma(A)\right\rangle$. If $\varphi\left(G_{1}\right)$ is non-zero, then, as before, there are finitely many vertices and we get a surjection from $K$ onto $\mathbb{F}_{\infty}$ by killing all the vertex groups.

If $\varphi\left(G_{1}\right)=0$, the image of $\varphi$ is generated by $\varphi(t)$. The valence of vertices in $T / K$ is the same as that in $T / G$, which is 2 , so $T / K$ is a line. Denote its edges $e(i)$, for $i \in \mathbb{Z}$. The vertex stabilizers are conjugates of $G_{1}$. To map $K$ onto $\mathbb{F}_{\infty}$, one should not kill all edge groups $G_{e(i)}$ because vertex groups might be killed too, but one can kill every other edge group $G_{e(2 i)}$. This maps $K$ onto an infinite free product, and we check that each factor may be mapped onto $\mathbb{Z}$.

Such a factor has the form $H=B *\langle c\rangle D$, where each group $B, D$ is isomorphic to the quotient of $G_{1}$ by an incident edge group $\left(G_{e(2 i)}\right.$ or $\left.G_{e(2 i+2)}\right)$. Again, 
since the first Betti number of $G_{1}$ is at least 2, there exist epimorphisms $\rho: B \rightarrow \mathbb{Z}$ and $\tau: D \rightarrow \mathbb{Z}$. If $\rho(c)=\tau(c)=0$, then $H$ maps onto $\mathbb{F}_{2}$. Otherwise, define a non-zero map from $H$ to $\mathbb{Z}$ as $\tau(c)$ times $\rho$ on $B$ and $\rho(c)$ times $\tau$ on $D$.

\section{Mapping tori of polynomially growing free group automorphisms}

Given $\alpha \in \operatorname{Aut}\left(\mathbb{F}_{n}\right)$, we denote by $G_{\alpha}$ the mapping torus

$$
G_{\alpha}=\mathbb{F}_{n} \rtimes_{\alpha} \mathbb{Z}=\left\langle\mathbb{F}_{n}, t \mid t^{-1} g t=\alpha(g)\right\rangle .
$$

Up to isomorphism, it only depends on the outer automorphism $\Phi \in \operatorname{Out}\left(\mathbb{F}_{n}\right)$ represented by $\alpha$, so we often write $G_{\Phi}$ rather than $G_{\alpha}$.

Let $\varphi: G_{\alpha} \rightarrow \mathbb{Z}$ be the map sending $\mathbb{F}_{n}$ to 0 and $t$ to 1 . We say that $\varphi$ (and any multiple of it) is a fibration with fiber $\mathbb{F}_{n}$ and monodromy $\alpha$ (or $\Phi$ ).

Recall that $\alpha$ (or $\Phi$ ) is polynomially growing if, for any $g \in \mathbb{F}_{n}$, the length of $\alpha^{k}(g)$ grows polynomially.

Remark 5.1. The automorphism $\alpha$ has a well-defined degree of polynomial growth $d(\alpha)$, which is the maximal degree of growth of the length, with respect to some fixed word metric on $\mathbb{F}_{n}$, of the shortest conjugate of $\alpha^{k}(g)$. Macura [24] has shown that $d(\alpha)$ is a quasi-isometry invariant of $G_{\alpha}$. In particular, if $\alpha \in \operatorname{Aut}\left(\mathbb{F}_{n}\right)$ and $\beta \in \operatorname{Aut}\left(\mathbb{F}_{m}\right)$ yield isomorphic mapping tori $G_{\alpha} \simeq G_{\beta}$, then $d(\alpha)=d(\beta)$.

The main result of this section is the following:

Theorem 5.2. Let $G=G_{\alpha}=\mathbb{F}_{n} \rtimes_{\alpha} \mathbb{Z}$ for $\alpha \in \operatorname{Aut}\left(\mathbb{F}_{n}\right)$ polynomially growing, with $n \geq 2$. There exist elements $t_{1}, \ldots, t_{n-1}$ in $G \backslash \mathbb{F}_{n}$ (not necessarily distinct), such that

$$
\Sigma(G)=-\Sigma(G)=\bigcap_{i} S\left(G, t_{i}\right)^{c} .
$$

More precisely, for each non-zero $\varphi: G \rightarrow \mathbb{R}$ the following hold:

- If some $\varphi\left(t_{i}\right)$ is 0 , then $[\varphi] \notin \Sigma(G)$. If $[\varphi]$ is discrete, then $\operatorname{ker} \varphi$ virtually surjects onto $\mathbb{F}_{\infty}$.

- If no $\varphi\left(t_{i}\right)$ is 0 , then $[\varphi] \in \Sigma(G) \cap-\Sigma(G)$ and $\operatorname{ker} \varphi$ is free. If $[\varphi]$ is discrete, then $\operatorname{ker} \varphi$ has finite rank.

Corollary 5.3. If the first Betti number of $G$ is at least 2, then

$$
S(G) \backslash \Sigma(G)=\bigcup_{i} S\left(G, t_{i}\right)
$$

is a non-empty collection of rationally defined great subspheres; in particular, $\Sigma(G) \neq S(G)$. 
Using Remark 2.11, we also have:

Corollary 5.4. A mapping torus of a polynomially growing free group automorphism does not admit a decomposition as a strictly ascending HNN-extension with finitely generated base group.

These results do not hold for exponentially growing automorphisms, as evidenced by the group $G$ constructed by Leary-Niblo-Wise [20]. It is the mapping torus of an automorphism of $\mathbb{F}_{3}$, and also of an injective, non-surjective, endomorphism of $\mathbb{F}_{2}$. It does not satisfy $\Sigma(G)=-\Sigma(G)$, and there exists a discrete $[\varphi]$ such that $\operatorname{ker} \varphi$ is a strictly increasing union of 2-generated subgroups, hence is infinitely generated but does not virtually surject onto $\mathbb{F}_{\infty}$.

\subsection{UPG automorphisms}

Recall the natural map $\tau$ : $\operatorname{Out}\left(\mathbb{F}_{n}\right) \rightarrow \mathrm{GL}_{n}(\mathbb{Z})$ recording the action of automorphisms on the abelianization of $\mathbb{F}_{n}$.

Definition 5.5 ([2]). Let UPG $\left(\mathbb{F}_{n}\right)$ be the set of polynomially growing elements $\Phi \in \operatorname{Out}\left(\mathbb{F}_{n}\right)$ that have unipotent image in $\mathrm{GL}_{n}(\mathbb{Z})$, and $\widehat{\mathrm{UPG}}\left(\mathbb{F}_{n}\right)$ is the preimage of $\operatorname{UPG}\left(\mathbb{F}_{n}\right)$ in $\operatorname{Aut}\left(\mathbb{F}_{n}\right)$.

Remark 5.6. If $n \geq 1$ and $\Phi$ is UPG, then 1 is an eigenvalue of $\tau(\Phi)$, and this guarantees that $G_{\Phi}$ has first Betti number at least 2. In particular, given any $g \in G_{\Phi}$, there is a non-trivial map from $G_{\Phi}$ to $\mathbb{Z}$ killing $g$.

Lemma 5.7 ([2, Corollary 5.7.6]). Every polynomially growing element of the group $\operatorname{Out}\left(\mathbb{F}_{n}\right)$ has a power in $\mathrm{UPG}\left(\mathbb{F}_{n}\right)$.

Lemma 5.8. If $\Phi \in \operatorname{UPG}\left(\mathbb{F}_{n}\right)$ has finite order, then it is the identity.

Proof. Being unipotent and of finite order, $\tau(\Phi)$ is trivial, so $\Phi \in \operatorname{ker} \tau$. But $\operatorname{ker} \tau$ is torsion-free [1].

The other relevant fact about unipotent polynomially growing automorphisms that we need is the existence of invariant free splittings of $\mathbb{F}_{n}$.

Proposition 5.9. Let $n \geq 2$. Every $\Phi \in \mathrm{UPG}\left(\mathbb{F}_{n}\right)$ has a representative $\alpha \in \operatorname{Aut}\left(\mathbb{F}_{n}\right)$ such that one of the following holds:

(1) There exists a non-trivial $\alpha$-invariant splitting $\mathbb{F}_{n}=B_{1} * B_{2}$, so $\alpha=\alpha_{1} * \alpha_{2}$ with $\alpha_{i}=\alpha_{\mid B_{i}} \in \operatorname{Aut}\left(B_{i}\right)$.

(2) There exists a non-trivial splitting $\mathbb{F}_{n}=B_{1} *\langle x\rangle$, where $B_{1} \simeq \mathbb{F}_{n-1}$ is $\alpha$-invariant, and $\alpha(x)=x u$ with $u \in B_{1}$. We denote $\alpha_{1}=\alpha_{\mid B_{1}}$. 
Proof. This is a consequence of Bestvina, Feighn and Handel's train track theory for free group automorphisms. By [2, Theorem 5.1.8], there exists a graph $\Gamma$ with valence at least 2 and fundamental group $\mathbb{F}_{n}$, a homotopy equivalence $f: \Gamma \rightarrow \Gamma$ inducing $\Phi$ on the fundamental group, and a filtration

$$
\emptyset=\Gamma_{0} \subset \Gamma_{1} \subset \cdots \subset \Gamma_{k}=\Gamma
$$

satisfying several properties. Relevant for us are that $f$ fixes the vertices of $\Gamma$, for every $i$ the stratum $\Gamma_{i} \backslash \Gamma_{i-1}$ is a single edge $E_{i}$, and $f\left(E_{i}\right)=E_{i} u_{i}$ where $u_{i}$ is a loop in $\Gamma_{i-1}$.

The proposition is proven by considering the topmost stratum $E_{k}$ of the filtration. If $E_{k}$ is a separating edge, then we are in case (1), and $B_{1}$ and $B_{2}$ are the fundamental groups of the two components of $\Gamma_{k-1}$. If $E_{k}$ is a non-separating edge, then we are in case (2), and $B_{1}$ is the fundamental group of $\Gamma_{k-1}$.

Lemma 5.10. If $\Phi \in \mathrm{UPG}\left(\mathbb{F}_{n}\right)$, then $G_{\Phi}$ admits a good $\mathbb{Z}$-hierarchy with $\mathbb{Z}^{2}$ leaves and $n-1$ splittings. Moreover, each edge group in the hierarchy has trivial intersection with the fiber $\mathbb{F}_{n}$, and each vertex group has first Betti number at least 2.

Proof. The lemma is proved by induction on $n$. If $n=1$, then $G_{\Phi}=\mathbb{Z}^{2}$ and we take the trivial hierarchy, which has $n-1=0$ splittings. Otherwise, we construct the first splitting in the hierarchy as follows. Apply Proposition 5.9. In the first case, we write $G_{\alpha}=G_{\alpha_{1}} *\langle t\rangle G_{\alpha_{2}}$. In the second case, we have

$$
G_{\alpha}=\left\langle B_{1}, x, t \mid t^{-1} b t=\alpha_{1}(b), t^{-1} x t=x u\right\rangle=\left\langle G_{\alpha_{1}}, x \mid x^{-1} t x=t u^{-1}\right\rangle,
$$

and we consider the $\mathrm{HNN}$-extension $G_{\alpha}=G_{\alpha_{1}} * \mathbb{Z}$ (which is not ascending since $G_{\alpha_{1}}$ is the mapping torus of an automorphism of $B_{1} \cong \mathbb{F}_{n-1}$, so it is not cyclic).

By the induction hypothesis, each $G_{\alpha_{i}}$ admits a good $\mathbb{Z}$-hierarchy with $\mathbb{Z}^{2}$ leaves. Take the hierarchy for $G$ consisting of the splitting we just constructed, and then the hierarchies for the $G_{\alpha_{i}}$. In the amalgamated product case the number of splittings is $1+\left(\operatorname{rank}\left(B_{1}\right)-1\right)+\left(\operatorname{rank}\left(B_{2}\right)-1\right)=n-1$. In the HNN-extension case the number of splittings is $1+\left(\operatorname{rank}\left(B_{1}\right)-1\right)=n-1$.

The claim about edge groups is clear from the way the hierarchy is constructed, and the Betti number claim follows from Remark 5.6: since $B_{i}$ as in Proposition 5.9 is an $\alpha$-invariant free factor of $\mathbb{F}_{n}$, the restriction $\alpha_{i}$ is UPG.

\subsection{Proof of Theorem 5.2}

We first note that it suffices to prove the theorem for a power of $\alpha$ :

Lemma 5.11. If the theorem is true for $\alpha^{p}$ with $p \geq 2$, then it is true for $\alpha$. 
Proof. Note that $G_{\alpha^{p}}=\left\langle\mathbb{F}_{n}, t^{p}\right\rangle$ is contained in $G_{\alpha}$ with finite index, so $\operatorname{ker} \varphi_{\mid G_{\alpha} p}$ is contained in $\operatorname{ker} \varphi$ with finite index. By [29, Proposition B1.11], we have that $\left[\varphi_{\mid G_{\alpha} p}\right] \in \Sigma\left(G_{\alpha^{p}}\right)$ if and only if $[\varphi] \in \Sigma\left(G_{\alpha}\right)$.

Applying the theorem to $\alpha^{p}$ yields elements $t_{i}$ in $G_{\alpha^{p}} \backslash \mathbb{F}_{n}$, hence in $G_{\alpha} \backslash \mathbb{F}_{n}$. These elements also work for the automorphism $\alpha$. If $\operatorname{ker} \varphi_{\mid G_{\alpha} p}$ virtually maps onto $\mathbb{F}_{\infty}$, so does $\operatorname{ker} \varphi$. If $\operatorname{ker} \varphi_{\mid G_{\alpha} p}$ is free, so is $\operatorname{ker} \varphi$ because it is torsion-free and virtually free [30].

By Lemmas 5.11 and 5.7 we may assume that $\Phi$ is UPG. Take the hierarchy for $G$ provided by Lemma 5.10. Define $t_{1}, \ldots, t_{n-1}$ as generators of the edge groups $A_{i}$ which occur in the hierarchy.

Corollary 4.3 says that $\Sigma(G)=\bigcap_{i \in \mathcal{l}} S\left(G, A_{i}\right)^{c}$, so $[\varphi] \in \Sigma(G)$ if and only if no $\varphi\left(t_{i}\right)$ equals 0 . The elements $t_{i}$ do not belong to $\mathbb{F}_{n}$ by the "moreover" of Lemma 5.10.

If $[\varphi] \in \Sigma(G)^{c}$ is discrete, then Theorem 4.4 says $\operatorname{ker} \varphi$ surjects onto $\mathbb{F}_{\infty}$.

If $[\varphi] \in \Sigma(G)$, then Theorem 4.4 says $\operatorname{ker} \varphi$ is a free product whose factors are isomorphic to groups $\operatorname{ker} \varphi_{\mid H_{j}}$, where $\left\{H_{j} \mid j \in \mathcal{A}\right\}$ are the leaf groups of the hierarchy and $\operatorname{ker} \varphi_{\mid H_{j}} \neq H_{j}$. In this case, the leaf groups are $\mathbb{Z}^{2}$, so $\operatorname{ker} \varphi_{\mid H_{j}}$ is either 1 or $\mathbb{Z}$. Thus, $\operatorname{ker} \varphi$ is a free group, and if $[\varphi]$ is discrete then $\operatorname{ker} \varphi$ has finite rank.

This completes the proof of Theorem 5.2.

Remark 5.12. Strebel [29, Problem B1.13] notes that $\Sigma$ is well behaved upon passing to finite index subgroups, and asks for examples in which calculating $\Sigma(G)$ directly is difficult, but $G$ contains a finite index subgroup $H$ for which $\Sigma(H)$ can be computed. Mapping tori of polynomially growing free group automorphisms provide such examples.

\subsection{Examples}

Theorem 5.2 claims that $S(G) \backslash \Sigma(G)=\bigcup_{i} S\left(G, t_{i}\right)$ is the union of $n-1$ great subspheres. These subspheres are not necessarily distinct. For instance, the mapping torus of the trivial automorphism of $\mathbb{F}_{n}$ is isomorphic to $\mathbb{F}_{n} \times \mathbb{Z}$; in this case the complement of $\Sigma(G)$ is a single sphere $S(G, t)$, with $t$ a generator of the $\mathbb{Z}$ factor. On the other hand, the following example shows that $n-1$ distinct spheres may be required.

Example 5.13. Take a graph $\Gamma$ that is a circle with vertices $v_{0}, \ldots, v_{n-1}$ and edges $b_{i}=\left[v_{i-1}, v_{i}\right]$, with indices modulo $n$. At each vertex $v_{i}$ add a loop $a_{i}$. Define a relative train track map on this graph fixing each $a_{i}$ and sending each $b_{i}$ to $b_{i} a_{i}$. This induces an outer automorphism $\Phi$ of the fundamental group $\mathbb{F}_{n}$ of the graph, and we let $G=G_{\Phi}$. Choosing $v_{i}$ as a basepoint for $\Gamma$ defines 
a representative $\alpha_{i} \in \operatorname{Aut}\left(\mathbb{F}_{n}\right)$ of $\Phi$, and there are associated stable letters $t_{i}$ such that $G=\mathbb{F}_{n} \rtimes_{\alpha_{i}} \mathbb{Z}=\left\langle\mathbb{F}_{n}, t \mid t_{i} g t_{i}^{-1}=\alpha_{i}(g)\right\rangle$. These are the elements featured in Theorem 5.2.

The mapping torus can be written as a graph of groups with underlying graph a circle with edges corresponding to the edges $b_{i}$. The vertex stabilizers are $\mathbb{Z}^{2}=\left\langle a_{i}, t_{i}\right\rangle$, because the loops $a_{i}$ are fixed by the automorphism. The edge stabilizers are infinite cyclic. The edge corresponding to $b_{i}$ amalgamates $t_{i-1}$ to $t_{i} a_{i}^{-1}$. Since the images of $a_{1}, a_{1} a_{2}, \ldots, a_{1} \cdots a_{n-2}$ in the abelianization of $G$ are linearly independent, the spheres $S\left(G, t_{i}\right)$ are distinct.

Note the example is a linearly growing automorphism, so the number of spheres does not correlate to degree of growth.

One might guess that the rank of the fiber blows up near $\Sigma(G)^{c}$. The following example shows that this is not necessarily true.

Example 5.14. Consider the mapping torus $G=\mathbb{F}_{n} \times \mathbb{Z}=\left\langle x_{1}, \ldots, x_{n}\right\rangle \times\langle z\rangle$. Take positive, coprime integers $p$ and $q$, and define $\varphi_{p, q}: G \rightarrow \mathbb{Z}$ by $\varphi_{p, q}\left(x_{i}\right)=p$ and $\varphi_{p, q}(z)=q$. Projection to the $\mathbb{F}_{n}$ factor is injective on $\operatorname{ker} \varphi_{p, q}$, and the image is an index $q$ subgroup of $\mathbb{F}_{n}$ (consisting of all elements with exponent sum divisible by $q$ ), so $\operatorname{ker} \varphi_{p, q}$ has rank $q(n-1)+1$. It is a fiber of a fibration (whose monodromy has order $q$ ). Now $\left[\varphi_{p, q}\right]=\left[\varphi_{1, q / p}\right]$, so if we fix any $q$ and let $p$ grow, the sequence $\left(\left[\varphi_{p, q}\right]\right)_{p} \subset \Sigma(G)$ converges to $\left[\varphi_{1,0}\right] \in \Sigma(G)^{c}$ through fibrations with constant fiber rank.

\section{The rank of the fiber}

Let $G=G_{\alpha}=\mathbb{F}_{n} \rtimes_{\alpha} \mathbb{Z}$ be the mapping torus of a polynomially growing automorphism $\alpha \in \operatorname{Aut}\left(\mathbb{F}_{n}\right)$, with $n \geq 2$. By Theorem 5.2, there exist elements $t_{i}$, $i=1, \ldots, n-1$, in $G \backslash \mathbb{F}_{n}$ (not necessarily distinct), such that, given any surjection $\varphi: G_{\alpha} \rightarrow \mathbb{Z}$, either some $\varphi\left(t_{i}\right)$ is 0 and $\operatorname{ker} \varphi$ is infinitely generated, or no $\varphi\left(t_{i}\right)$ is 0 and $\operatorname{ker} \varphi$ is free of finite rank.

The main result of this section is the following.

Theorem 6.1. Let $\alpha \in \operatorname{Aut}\left(\mathbb{F}_{n}\right)$ be polynomially growing, and let $G_{\alpha}$ be its mapping torus. There exist elements $t_{1}, \ldots, t_{n-1} \in G_{\alpha} \backslash \mathbb{F}_{n}$ as in Theorem 5.2 such that, if $\varphi: G_{\alpha} \rightarrow \mathbb{Z}$ is a surjection such that no $\varphi\left(t_{i}\right)=0$, the rank of the free group $\operatorname{ker} \varphi$ is

$$
r=1+\frac{1}{k} \sum_{i=1}^{n-1}\left|\varphi\left(t_{i}\right)\right|
$$

with $k$ the least positive integer such that $\alpha^{k} \in \widehat{\mathrm{UPG}}\left(\mathbb{F}_{n}\right)$. 
Example 6.2. If $G=\mathbb{F}_{n} \times \mathbb{Z}$ is as in Example 5.14, one may take all $t_{i}$ equal to $z$, and $r=1+(n-1)|\varphi(z)|$. In particular, the rank of $\operatorname{ker} \varphi_{p, q}$ is $1+(n-1) q$. See Section 7 for groups which are virtually $\mathbb{F}_{n} \times \mathbb{Z}$.

Corollary 6.3. Fibers with UPG monodromy have minimal rank.

Proof. Suppose $\alpha \in \operatorname{Aut}\left(\mathbb{F}_{n}\right)$ is UPG. If $\varphi: G_{\alpha} \rightarrow \mathbb{Z}$ has kernel $\mathbb{F}_{r}$, we have

$$
r=1+\sum_{i=1}^{n-1}\left|\varphi\left(t_{i}\right)\right| \geq n
$$

since $k=1$ and $\left|\varphi\left(t_{i}\right)\right| \geq 1$ for all $i$.

Corollary 6.4. If $G$ is the mapping torus of a polynomially growing automorphism of a non-abelian free group, and the first Betti number of $G$ is at least 2, then $G$ admits fibrations with fibers of unbounded rank.

Proof. Since the Betti number is $\geq 2$, we may find $\varphi: G \rightarrow \mathbb{Z}$ with no $\varphi\left(t_{i}\right)$ equal to 0 , and $\varphi\left(t_{1}\right)$ arbitrarily large.

The rank of the fiber can alternatively be calculated as the degree of the Alexander polynomial of $G$ relative to $\varphi$ (see [8,27]). McMullen [25] determined the Alexander invariants for 3-manifolds. Button [8] gives an algorithm for computing the relative Alexander polynomial of a group admitting a deficiency 1 presentation, including mapping tori of free group automorphisms, using Fox Calculus. By generalizing McMullen's arguments, he is able to give lower bounds for the Betti number of the kernel, in particular proving Lemma 6.4 without the polynomial growth hypothesis, using the fact that the Alexander polynomial of the mapping torus is non-constant [8, Theorem 3.4].

We prove Theorem 6.1 without using the Alexander invariants by computing the kernel from the hierarchical structure of the mapping torus.

Proof of Theorem 6.1. We will consider restrictions of $\varphi$ to subgroups. Since they are not necessarily surjective, we rewrite the formula in a homogeneous way.

For $\varphi$ real-valued but with cyclic image, and $H<G_{\alpha}$, define $\left[G_{\alpha}: H\right]_{\varphi}$ as the index $\left[\varphi\left(G_{\alpha}\right): \varphi(H)\right]$. We write $\left[G_{\alpha}: t_{i}\right]_{\varphi}$ rather than $\left[G_{\alpha}:\left\langle t_{i}\right\rangle\right]_{\varphi}$. With this notation, we have to prove

$$
r-1=\frac{1}{k} \sum_{i=1}^{n-1}\left[G_{\alpha}: t_{i}\right]_{\varphi} .
$$

First consider the UPG case. As in the proof of Theorem 5.2, we argue by induction on the number of splittings in a hierarchy provided by Lemma 5.10. In the 
proof of Theorem 4.4 we showed that $K=\operatorname{ker} \varphi$ decomposes as the fundamental group of a graph of groups $\Gamma_{K}$ with trivial edge groups. The rank of $K$ is therefore the first Betti number of the graph $\Gamma_{K}$ plus the sum of the ranks of the vertex groups. The ranks of the vertex groups are computed inductively.

The proposition is true if the hierarchy is trivial. If not, we consider the first splitting in the hierarchy. We denote by $t_{1}$ a generator of the edge group of this splitting, and by $t_{2}, \ldots, t_{n-1}$ generators associated to the other edge groups of the hierarchy.

There are two cases. First consider the HNN case $G_{\alpha}=G_{1} *\left\langle t_{1}\right\rangle$. By Lemma 4.5, $\Gamma_{K}$ has $\left[G_{\alpha}: G_{1}\right]_{\varphi}$ vertices, each carrying a free group of rank $1+\sum_{i=2}^{n-1}\left[G_{1}: t_{i}\right]_{\varphi}$ by the induction hypothesis, and $\left[G_{\alpha}: t_{1}\right]_{\varphi}$ edges. The first Betti number of the graph is

$$
1-\left[G_{\alpha}: G_{1}\right]_{\varphi}+\left[G_{\alpha}: t_{1}\right]_{\varphi},
$$

so

$$
r=1-\left[G_{\alpha}: G_{1}\right]_{\varphi}+\left[G_{\alpha}: t_{1}\right]_{\varphi}+\left[G_{\alpha}: G_{1}\right]_{\varphi}\left(1+\sum_{i=2}^{n-1}\left[G_{1}: t_{i}\right]_{\varphi}\right),
$$

yielding

$$
r=1+\left[G_{\alpha}: t_{1}\right]_{\varphi}+\sum_{i=2}^{n-1}\left[G_{\alpha}: t_{i}\right]_{\varphi}=1+\sum_{i=1}^{n-1}\left[G_{\alpha}: t_{i}\right]_{\varphi} .
$$

The computation in the amalgam case is similar, except that there are two types of vertices.

If $\alpha$ is not UPG, let $G_{k}$ denote $G_{\alpha^{k}}$, which is an index $k$ subgroup of $G_{\alpha}$. Let $\varphi_{k}$ be the restriction of $\varphi$ to $G_{k}$, and let $r_{k}$ the rank of $\operatorname{ker} \varphi_{k}$. We may take the same elements $t_{i}$ for $\alpha$ and $\alpha^{k}$ (see the proof of Lemma 5.11), and

$$
\left[\operatorname{ker} \varphi: \operatorname{ker} \varphi_{k}\right](r-1)=r_{k}-1=\sum_{i=1}^{n-1}\left[G_{k}: t_{i}\right]_{\varphi} .
$$

Considering the exact sequence

$$
1 \rightarrow \operatorname{ker} \varphi \rightarrow G_{\alpha} \rightarrow \mathbb{R}
$$

and restricting to $G_{k}$, which has index $k$ in $G_{\alpha}$, we see that

$$
k=\left[G_{\alpha}: G_{k}\right]_{\varphi}\left[\operatorname{ker} \varphi: \operatorname{ker} \varphi_{k}\right],
$$

so multiplying by $\left[G_{\alpha}: G_{k}\right]_{\varphi}$ yields

$$
k(r-1)=\left[G_{\alpha}: G_{k}\right]_{\varphi} \sum_{i=1}^{n-1}\left[G_{k}: t_{i}\right]_{\varphi}=\sum_{i=1}^{n-1}\left[G_{\alpha}: t_{i}\right]_{\varphi} .
$$




\section{Finite order automorphisms and GBS groups with center}

We now suppose that $G$ is the mapping torus of a finite order element $\Phi \in \operatorname{Out}\left(\mathbb{F}_{n}\right)$, for some $n \geq 2$. By [23, Proposition 4.1], this is equivalent to $G$ being a GBS group with non-trivial center, and being non-elementary (i.e. not isomorphic to $\mathbb{Z}$, $\mathbb{Z}^{2}$, or the Klein bottle group). It has a finite index subgroup isomorphic to $\mathbb{F}_{m} \times \mathbb{Z}$ for some $m \geq 2$. The main results of this section (Theorem 7.3 and Theorem 7.4) will give quantitative versions of these facts.

We refer to $[12,13,22,23]$ for basic facts about GBS groups. In terms of the modular map $\Delta: G \rightarrow \mathbb{Q}^{*}$ of Section 3, a non-elementary GBS group has nontrivial center if and only if $\Delta$ is trivial.

The group $G$ acts on a tree $T$ with infinite cyclic edge and vertex stabilizers (as usual, we assume that the action of $G$ on $T$ is minimal). Elements of $G$ fixing a point in $T$ are called elliptic. The set of elliptic elements does not depend on the choice of $T$, and consists of finitely many conjugacy classes of cyclic subgroups (because $T / G$ is a finite graph).

The center of $G$, denoted by $Z$, is infinite cyclic and equals the set of elements acting as the identity on $T$ (see [22, Proposition 2.5]). This implies that, if a subgroup $H<G$ acts on $T$ minimally, in particular if $H$ has finite index, or is normal and non-central, its centralizer is equal to $Z$.

The quotient $G / Z$ acts on the tree $T$ with finite stabilizers, so is virtually free. If $L<G / Z$ is free, its preimage is isomorphic to $L \times \mathbb{Z}$ because it is a central $\mathbb{Z}$-by-free extension.

Definition 7.1. Given a non-elementary GBS group $G$ with non-trivial center $Z$, we define numbers $\kappa$ and $\epsilon$ as follows:

- $\kappa$ is the lcm of orders of torsion elements of $G / Z$,

- $\epsilon+1$ is the smallest rank of a free subgroup of finite index $L<G / Z$.

The group $G / Z$ is the fundamental group of a finite graph of groups whose vertex groups are finite cyclic groups, and $\kappa$ is the lcm of their orders. If $L \subset G / Z$ is a free subgroup of finite index, it is well known that its index is divisible by $\kappa$ (each vertex group acts freely on the set of cosets modulo $L$ ), and $\kappa$ is the smallest index of a free subgroup of $G / Z$ (Serre [28, Section II.2.6, Lemma 10] defines a map of $G / Z$ into permutations of $\kappa$ elements so that the vertex groups act freely; the preimage of any point stabilizer is a free subgroup of index $\kappa$ ).

It follows that there exist free subgroups of index $p \kappa$ for every $p \geq 1$. An Euler characteristic argument shows that the rank is $p \epsilon+1$ if the index is $p \kappa$.

Going back to $G$, we may view $\kappa$ as the smallest integer such that $a^{\kappa} \in Z$ for every elliptic element $a \in G$. We also have the following lemma. 
Lemma 7.2. Let $E \subset G$ be the (normal) subgroup generated by all elliptic elements. If $\varphi: G \rightarrow \mathbb{R}$ is a homomorphism such that $\varphi(Z) \neq 0$, then

$$
\kappa=[\varphi(E): \varphi(Z)] .
$$

Since any elliptic element has a power in $Z$, the restriction of $\varphi$ to $E$ is unique up to a multiplicative constant.

Proof. Let $z$ be a generator of $Z$. It acts as the identity on $T$, so if $a$ generates a vertex stabilizer there exists $\kappa_{a}$ such that $z=a^{\kappa_{a}}$. The number $\kappa$ is the lcm of the numbers $\kappa_{a}$, while $\varphi(E)$ is generated by the numbers $\varphi(z) / \kappa_{a}$. The lemma follows.

Theorem 7.3. Let $G$ be a non-elementary GBS group $G$ with non-trivial center $Z$. Given positive integers $k$ and $n$, the following are equivalent:

(1) $(k, n-1)$ is an integral multiple of $(\kappa, \epsilon)$.

(2) $G / Z$ has a subgroup of index $k$ isomorphic to $\mathbb{F}_{n}$.

(3) $G$ has a subgroup $G_{0}$ of index $k$ isomorphic to $\mathbb{F}_{n} \times \mathbb{Z}$, with $Z \subset G_{0}$.

(4) $G$ has a subgroup $G_{0}$ isomorphic to $\mathbb{F}_{n} \times \mathbb{Z}$ whose index is finite and equal to $k\left[Z: G_{0} \cap Z\right]$.

Proof. We already know that (1) and (2) are equivalent. For $p \geq 1$, let $L \simeq \mathbb{F}_{p \epsilon+1}$ have index $p \kappa$ in $G / Z$. Its preimage in $G$ contains $Z$, has index $p \kappa$, and is isomorphic to $\mathbb{F}_{p \epsilon+1} \times \mathbb{Z}$. This proves that (1) implies (3), and (3) trivially implies (4). Conversely, if $G_{0}$ is as in (4), the $\mathbb{Z}$ factor is contained in $Z$ (it centralizes $G_{0}$, which acts minimally on $T$, so it acts trivially on $T$ ). The image of $G_{0}$ in $G / Z$ is free of rank $n$ and has index $k=\left[G: G_{0}\right] /\left[Z: G_{0} \cap Z\right]$, so (2) holds.

We now consider fibrations of $G$.

Theorem 7.4. Let $G$ be a non-elementary GBS group $G$ with non-trivial center. Let $k$ and $n$ be positive integers.

(1) If the first Betti number of $G$ is 1 , there exists an element $\Phi \in \operatorname{Out}\left(\mathbb{F}_{n}\right)$ of order $k$ such that $G \simeq G_{\Phi}$ if and only if $(k, n-1)=(\kappa, \epsilon)$.

(2) If the first Betti number of $G$ is at least 2, there exists an element $\Phi \in \operatorname{Out}\left(\mathbb{F}_{n}\right)$ of order $k$ such that $G \simeq G_{\Phi}$ if and only if $(k, n-1)$ is an integral multiple of $(\kappa, \epsilon)$.

The first Betti number $b_{1}(G)$ of $G$ is equal to $1+b_{1}(\Gamma)$, with $\Gamma$ the quotient graph $T / G$ (see [22, Proposition 3.3]), and $G / E$ is free of rank $b_{1}(\Gamma)$ (this is a general fact about graphs of groups). In particular, $b_{1}(G)=1$ is equivalent to $\Gamma$ being a tree, and to $E=G$. 
Corollary 7.5. If the first Betti number of $G$ is at least 2, then ranks of fibers are an arithmetic progression: there is an exact sequence $1 \rightarrow \mathbb{F}_{n} \rightarrow G \rightarrow \mathbb{Z} \rightarrow 1$ if and only if $n$ is of the form $p \epsilon+1$ with $p \geq 1$.

Before proving the theorem, we note:

Lemma 7.6. If $G=G_{\Phi}$, with $\Phi \in \operatorname{Out}\left(\mathbb{F}_{n}\right)$ of order $k$, and $\varphi: G \rightarrow \mathbb{Z}$ is the associated fibration, then $\varphi(Z)=k \mathbb{Z}$ and $\varphi^{-1}(k \mathbb{Z}) \simeq \mathbb{F}_{n} \times \mathbb{Z}$.

Proof. Let $\alpha \in \operatorname{Aut}\left(\mathbb{F}_{n}\right)$ be a representative of $\Phi$, let $t$ be the associated stable letter, and let $z$ be a generator of $Z$. Writing $z=g t^{q}$ with $g \in \mathbb{F}_{n}$, we show $|q|=|k|$. Since $z$ centralizes $\mathbb{F}_{n}$, the automorphism $\alpha^{q}$ is inner, so $q$ is a multiple of $k$. On the other hand, since $\Phi$ has order $k$, some $h t^{k}$, with $h \in \mathbb{F}_{n}$, centralizes $\mathbb{F}_{n}$ hence belongs to $Z$ (because $\mathbb{F}_{n}$ is normal so acts minimally on $T$ ). This implies that $k$ is a multiple of $q$, and therefore $|q|=|k|$. The extension

$$
1 \rightarrow \mathbb{F}_{n} \rightarrow \varphi^{-1}(k \mathbb{Z}) \rightarrow k \mathbb{Z} \rightarrow 1
$$

is trivial because $\varphi^{-1}(k \mathbb{Z})$ contains $z^{ \pm 1}$.

Proof of Theorem 7.4. Suppose $G=G_{\Phi}$, with $\Phi \in \operatorname{Out}\left(\mathbb{F}_{n}\right)$ of order $k$. It follows from Lemma 7.6 that $\varphi^{-1}(k \mathbb{Z})$ is a subgroup of index $k$ isomorphic to $\mathbb{F}_{n} \times \mathbb{Z}$ and containing $Z$, so $(k, n-1)$ is a multiple of $(\kappa, \epsilon)$ by Theorem 7.3. If $b_{1}(G)=1$, we have seen that $E=G$, so $k=\kappa$ by Lemma 7.2. We have proved the "only if" direction in both assertions of the theorem.

For the converse, first recall how to construct maps $\varphi: G \rightarrow \mathbb{R}$ with $\varphi(Z) \neq 0$ (see [22, Proposition 3.3]). View $G$ as the fundamental group of a graph of infinite cyclic groups. Consider a standard generating set, consisting of generators of vertex groups and stable letters; the number of stable letters is the first Betti number of the graph $\Gamma=T / G$, equal to $b_{1}(G)-1$. One first defines $\varphi$ on $E$ (as mentioned above, $\varphi_{\mid E}$ is unique up to scaling). One must then choose the image of the stable letters. Non-triviality of the center ensures that any choice yields a well-defined $\operatorname{map} \varphi: G \rightarrow \mathbb{R}$.

If $b_{1}(G) \geq 2$, there is at least one stable letter and one may construct an epimorphism $\varphi_{p}: G \rightarrow \mathbb{Z}$ such that $\varphi_{p}(E)=p \mathbb{Z}$ for any $p \geq 1$. If $b_{1}(G)=1$, there is no stable letter, $E=G$, and we only obtain $\varphi_{1}$.

We have $\varphi_{p}(Z)=p \kappa \mathbb{Z}$ by Lemma 7.2, and let $G_{p}$ be the preimage $\varphi_{p}^{-1}(p \kappa \mathbb{Z})$. It is a normal subgroup of index $p \kappa$ containing $Z$ and $\operatorname{ker} \varphi_{p}$.

Consider the projection $\pi: G \rightarrow G / Z$. The groups $G_{p}$ and $\operatorname{ker} \varphi_{p}$ have the same image, and $\pi$ is injective on $\operatorname{ker} \varphi_{p}$. Since every elliptic element $a \in G_{p}$ belongs to $Z$ (because $\langle a, Z\rangle$ is cyclic), the image of $G_{p}$ in $G / Z$ is torsion-free. It is a free subgroup of index $p \kappa$, and its rank is $p \epsilon+1$. We therefore have $\operatorname{ker} \varphi_{p} \simeq \mathbb{F}_{p \epsilon+1}$. By Lemma 7.6, the associated monodromy $\Phi$ has order $p \kappa$. 
Remark 7.7. The proof shows that $G$ has a normal subgroup of index $\kappa$ isomorphic to $\mathbb{F}_{n} \times \mathbb{Z}$ and containing $Z$, and $G / Z$ has a normal free subgroup of index $\kappa$.

One might ask whether the monodromies of fibrations with fiber of minimal rank $\epsilon+1$ for a fixed GBS group $G$ must be conjugate in $\operatorname{Out}\left(\mathbb{F}_{\epsilon+1}\right)$, or whether this must be true for fibrations in the same component of $\Sigma(G)$. The answer to both questions is 'no' once $n=\epsilon+1 \geq 4$.

For $n=2$, Bogopolski, Martino, and Ventura [5] show that mapping tori of $\left[\alpha_{1}\right],\left[\alpha_{2}\right] \in \operatorname{Out}\left(\mathbb{F}_{2}\right)$ are isomorphic if and only if $\left[\alpha_{1}\right]$ is conjugate to $\left[\alpha_{2}\right]$ or $\left[\alpha_{2}\right]^{-1}$ in $\operatorname{Out}\left(\mathbb{F}_{2}\right)$.

An example of Vikent'ev [32] shows this statement is not true in general for $n=3$, but Khramtsov [16] showed it is true for finite order outer automorphisms.

For $n=4$, Khramtsov [16] gives the following example. Let $G$ be the GBS group $\left\langle a, b, t \mid a^{4}=b^{2},[b, t]=1\right\rangle$. Consider $\varphi_{i}: G \rightarrow \mathbb{Z}$ defined by $\varphi_{1}(a)=1$, $\varphi_{1}(b)=2, \varphi_{1}(t)=0$ and $\varphi_{2}(a)=1, \varphi_{2}(b)=2$ and $\varphi(t)=1$. These surjections give fibrations of $G$ with fiber of rank 4 and monodromy of order 4 in $\operatorname{Out}\left(\mathbb{F}_{4}\right)$. The minimal fiber rank for $G$ is $n=\epsilon+1=4$, by Theorem 7.4, since $k=\kappa=4$. In this example, $\Sigma(G) \cong S^{1} \backslash S^{0}$ consists of classes of homomorphisms not killing the element $a$. Thus, $\left[\varphi_{1}\right]$ and $\left[\varphi_{2}\right]$ are in the same component of $\Sigma(G)$, since they both send $a$ to a positive number. Khramtsov gives an ad hoc argument to show the monodromies are non-conjugate. This can also be verified using a solution to the conjugacy problem for finite order elements of $\operatorname{Out}\left(\mathbb{F}_{n}\right)$. Such solutions follow from work of Krstić [18], and are explained in [17] or [19].

Acknowledgments. The authors thank Ilya Kapovich for his interest and encouragement, Goulnara Arzhantseva for translating Khramtsov's example, and Ralph Strebel for useful comments.

\section{Bibliography}

[1] G. Baumslag and T. Taylor, The centre of groups with one defining relator, Math. Ann. 175 (1968), 315-319.

[2] M. Bestvina, M. Feighn and M. Handel, The Tits alternative for $\operatorname{Out}\left(F_{n}\right)$. I: Dynamics of exponentially-growing automorphisms, Ann. of Math. (2) 151 (2000), no. 2, 517-623.

[3] R. Bieri, W. D. Neumann and R. Strebel, A geometric invariant of discrete groups, Invent. Math. 90 (1987), no. 3, 451-477.

[4] R. Bieri and R. Strebel, Geometric invariants for discrete groups, preprint (1992).

[5] O. Bogopolski, A. Martino and E. Ventura, The automorphism group of a free-bycyclic group in rank 2, Comm. Algebra 35 (2007), no. 5, 1675-1690. 
[6] M. R. Bridson and D. Groves, The quadratic isoperimetric inequality for mapping tori of free group automorphisms, Mem. Amer. Math. Soc. 203 (2010), no. 955.

[7] K.S. Brown, Trees, valuations, and the Bieri-Neumann-Strebel invariant, Invent. Math. 90 (1987), no. 3, 479-504.

[8] J. O. Button, Mapping tori with first Betti number at least two, J. Math. Soc. Japan 59 (2007), no. 2, 351-370.

[9] S. Dowdall, I. Kapovich and C. J. Leininger, Dynamics on free-by-cyclic groups, Geom. Topol. 19 (2015), no. 5, 2801-2899.

[10] S. Dowdall, I. Kapovich and C. J. Leininger, McMullen polynomials and Lipschitz flows for free-by-cyclic groups, preprint (2013), http://arXiv.org/abs/1310. $7481 \mathrm{v} 3$.

[11] M. Feighn and M. Handel, Mapping tori of free group automorphisms are coherent, Ann. of Math. (2) 149 (1999), no. 3, 1061-1077.

[12] M. Forester, On uniqueness of JSJ decompositions of finitely generated groups, Comment. Math. Helv. 78 (2003), no. 4, 740-751.

[13] M. Forester, Splittings of generalized Baumslag-Solitar groups, Geom. Dedicata 121 (2006), 43-59.

[14] F. Gautero and M. Lustig, The mapping-torus of a free group automorphism is hyperbolic relative to the canonical subgroups of polynomial growth, preprint (2007), http://arXiv.org/abs/0707.0822v2.

[15] R. Geoghegan, M. L. Mihalik, M. Sapir and D. T. Wise, Ascending HNN extensions of finitely generated free groups are Hopfian, Bull. Lond. Math. Soc. 33 (2001), no. 3 , 292-298.

[16] D. G. Khramtsov, Outer automorphisms of free groups (in Russian), in: Group-Theoretic Investigations, Akad. Nauk SSSR Ural. Otdel., Sverdlovsk (1990), 95-127.

[17] D. G. Khramtsov, Decidability of the conjugacy problem for finite subgroups of automorphism groups of free groups (in Russian), Algebra i Logika 34 (1995), no. 5, 558-606, 609; translation in Algebra Logic 34 (1995), no. 5, 311-337.

[18] S. Krstić, Actions of finite groups on graphs and related automorphisms of free groups, J. Algebra 124 (1989), no. 1, 119-138.

[19] S. Krstić, M. Lustig and K. Vogtmann, An equivariant Whitehead algorithm and conjugacy for roots of Dehn twist automorphisms, Proc. Edinb. Math. Soc. (2) 44 (2001), no. 1, 117-141.

[20] I. J. Leary, G. A. Niblo and D. T. Wise, Some free-by-cyclic groups, in: Groups St. Andrews 1997 in Bath. Vol. II, London Math. Soc. Lecture Note Ser. 261, Cambridge University Press, Cambridge (1999), 512-516.

[21] G. Levitt, R-trees and the Bieri-Neumann-Strebel invariant, Publ. Mat. 38 (1994), no. 1, 195-202. 
[22] G. Levitt, On the automorphism group of generalized Baumslag-Solitar groups, Geom. Topol. 11 (2007), 473-515.

[23] G. Levitt, Generalized Baumslag-Solitar groups: Rank and finite index subgroups, Ann. Inst. Fourier (Grenoble) 65 (2015), no. 2, 725-762.

[24] N. Macura, Detour functions and quasi-isometries, Q.J. Math. 53 (2002), no. 2, 207-239.

[25] C. T. McMullen, The Alexander polynomial of a 3-manifold and the Thurston norm on cohomology, Ann. Sci. Éc. Norm. Supér. (4) 35 (2002), no. 2, 153-171.

[26] G. Meigniez, Bouts d'un groupe opérant sur la droite. I: Théorie algébrique, Ann. Inst. Fourier (Grenoble) 40 (1990), no. 2, 271-312.

[27] J. W. Milnor, Infinite cyclic coverings, in: Conference on the Topology of Manifolds (Lansing 1967), Prindle, Weber \& Schmidt, Boston (1968), 115-133.

[28] J.-P. Serre, Trees, Springer Monogr. Math., Springer, Berlin, 2003.

[29] R. Strebel, Notes on the sigma invariants, preprint (2012), http://arXiv.org/ $\mathrm{abs} / 1204.0214 \mathrm{v} 2$.

[30] R. G. Swan, Groups of cohomological dimension one, J. Algebra 12 (1969), 585-610.

[31] W. P. Thurston, A norm for the homology of 3-manifolds, Mem. Amer. Math. Soc. 59 (1986), no. 339, i-vi and 99-130.

[32] R. A. Vikent'ev, On the isomorphism problem in the class of extensions of a free group by an infinite cyclic group, Sib. Math. J. 48 (2007), 428-429.

Received January 15, 2015.

\section{Author information}

Christopher H. Cashen, Fakultät für Mathematik, Universität Wien, Austria.

E-mail: christopher.cashen@univie.ac.at

Gilbert Levitt, Laboratoire de Mathématiques Nicolas Oresme, Université de Caen et CNRS (UMR 6139), France.

E-mail: levitt@unicaen.fr 